\title{
Preferencias de empleo de las estudiantes de doctorado en Ingeniería*
}

\section{Employment preferences for PhD students in Engineering}

\section{Jorge Enrique Celis Giraldo** \\ Norma Rocio Héndez Puerto Universidad Nacional de Colombia}

Recibido: 31 de julio de 2017 Revisado: 16 de octubre de 2017 Aceptado: 24 de enero de 2018

\section{Resumen}

Este artículo analiza las preferencias de empleo de las mujeres estudiantes de doctorado en Ingeniería. Estas preferencias oscilan entre la academia y la industria y se las compara con las de los estudiantes hombres. Trabajos previos de investigación sobre mujeres con doctorado se han enfocado en el clima laboral, las experiencias de acoso sexual, el impacto de los factores familiares en la trayectoria laboral y los ingresos salariales. El análisis está basado en información obtenida de una encuesta virtual aplicada a estudiantes de doctorado en ingeniería provenientes de una universidad en Colombia. Los resultados muestran que las estudiantes mujeres tienen mayor interés por espacios laborales en los cuales puedan realizar investigación y tienen una alta valoración por atributos laborales como la posibilidad de colaborar con otras instituciones /organizaciones. Una de las principales conclusiones del estudio es que 
las mujeres presentaron mayor inclinación por los atributos laborales que caracterizan a la academia mientras que los hombres evidencian una mayor por los relacionados con la industria y se mostraron menos interesados en optar por una carrera académica.

Palabras clave: educación y empleo; mujeres; programas de doctorado; ingeniería, grado de doctor; condiciones de empleo

\section{Abstract}

This paper analyses career preferences of women who are doing a Ph.D. program in engineering and compares them with those of men doing a Ph.D. program in engineering. Research on women with doctoral education has focused on exploring topics related to job climate, sexual harassment, the impact of family factors on job experience, employability, and salary earnings. The analysis is based on data collected from online survey administered to women doing a Ph.D. program in engineering at a university in Colombia. The results show that women have an interest in jobs in which they can do research as well as a high perception over job attributes, such collaboration with institutions/organization. One of the main conclusions of the study is that women show greater inclination for the work attributes of the academy while men show a greater tendency for those related to the industry and men are less interested in an academic career.

Keywords: education and job; women; PhD education; engineering; job conditions

\section{Introducción}

En las dos últimas décadas, la formación doctoral en Colombia ha experimentado una expansión significativa. Al observar las cifras correspondientes al periodo 2002-2014, se registra un incremento en el número de aspirantes interesados en cursar estudios de doctorado, de estudiantes matriculados y de graduados, así como en el número de programas de doctorado. A principios del Siglo XXI, en Colombia tan sólo 59 aspirantes se inscribieron a cursar programas de doctorado. Para el año 2014, este número fue de $\mathbf{2 . 2 8 0}$.

En Colombia, en el área de ingeniería se pasó de 47 aspirantes en el 2002 a 475. El país contaba con 350 estudiantes en el año 2002 y doce años después este número había ascendido a 8.941 siendo el área de Ciencias Sociales y Humanas la que más estudiantes concentraba (MEN-SNIES, 2016). En los programas de doctorado en Ingeniería hubo un crecimiento similar, se pasó de 108 estudiantes a 2.186 siendo ingeniería de sistemas y telemática la que registró la mayoría de estudiantes (MENSNIES, 2016). Los graduados en general crecieron también; pasaron de 32 en 2002 a 400 en 2014, y en las ingenierías pasaron de 4 a 94 (MEN-SNIES, 2016).

Las universidades ofrecían 64 programas de doctorado para el año 2002 y 285 en el 2014 y el área con mayor representatividad fue matemáticas y ciencias naturales. En ingeniería se ofrecían 6 programas en 2002 y 50 en 2014, siendo las disciplinas de ingeniería de sistemas y telemática e ingeniería industrial las que concentraban el mayor número de programas (MEN-SNIES, 2016). La expansión de la formación doctoral podría explicarse por las inversiones hechas en los últimos años para aumentar los créditos educativos (Acosta \& Celis, 2014).

Según cifras del Observatorio Colombia de Ciencia y Tecnología (OCYT), se otorgaban 239 créditos en el año 2007 y en el 2014 fueron 1.492. En 2007, del total de créditos otorgados $78,7 \%$ eran destinados para realizar estudios de doctorado en el exterior mientras que en el 2013 el 55,7 \% de los créditos fueron para realizar estudios en el país. El interés de tener un sistema de formación doctoral más robusto radica (Gómez \& Celis, 2009; Pineda, 2015) en que las universidades son vistas como actores 
fundamentales del Sistema Nacional de Ciencia, Tecnología e Innovación (SNCTI), pues son las encargadas no sólo de producir nuevo conocimiento dirigido a aportar al avance de las disciplinas, sino también de aumentar las comunidades científicas encargadas de fortalecer las capacidades endógenas del país para hacer investigación (Acosta \& Celis, 2014). No es coincidencia, en este marco, que las universidades concentren el $63,43 \%$ de grupos de investigación reconocidos por el Departamento Administrativo de Ciencia, Tecnología e Innovación (COLCIENCIAS) (OCYT, 2016). Pero también operan elementos de lucha simbólica en el campo de la educación superior.

Así pues, la investigación se ha convertido en un signo de distinción que diferencia unas universidades de otras, al punto que ésta es definida como uno de los principales factores que se tiene en cuenta a la hora de otorgar la acreditación a un programa académico como a una institución de educación superior (Celis, Camacho, Arenas, \& Duque, 2014; Gómez \& Celis, 2009). Por esto varias universidades han planteado como uno de los ejes estratégicos de desarrollo llegar a convertirse en universidades de clase mundial fundamentadas en la investigación y por eso, han decidido acrecentar la oferta de programas de doctorado y el número de estudiantes de doctorado (Altbach \& Jamil, 2011; Colmenares \& Celis, 2011; Salmi, 2009).

En Colombia, también se incrementó el número de profesionales con título de doctorado en la última década, en parte porque muchas universidades han venido exigiendo como requisito de ingreso a la carrera docente el título de doctorado (Acosta \& Celis, 2014; Rubio, Celis, \& Gómez, 2007). Como se puede observar en la Figura 1, Colombia pasó de tener 5.206 doctores en 2005 -con una representación del 26,8 \% de mujeres- a 11.181 en 2014 -con un peso porcentual del 32,1 \% para el caso de las mujeres- (OCYT, 2016). En el área de ingeniería y tecnología, de los 108 doctores que había en 2005 , el $15,4 \%$ eran mujeres mientras que para 2014 la representación femenina aumentó al 31,4 \% de un total de 1.181 doctores (ver Figura 2). Es importante señalar que el área de ingeniería y tecnología es el área de conocimiento que tuvo la mayor representación de mujeres entre los años 2005 y 2014; esto es, 16 \% más con respecto a áreas como ciencias médicas y ciencias humanas y sociales en las cuales el incremento fue tan solo del $1,2 \%$ y $7,4 \%$ respectivamente (OCYT, 2016).

Figura 1. Doctores en Colombia según género: 2005-2014. Fuente: OCYT (2016)

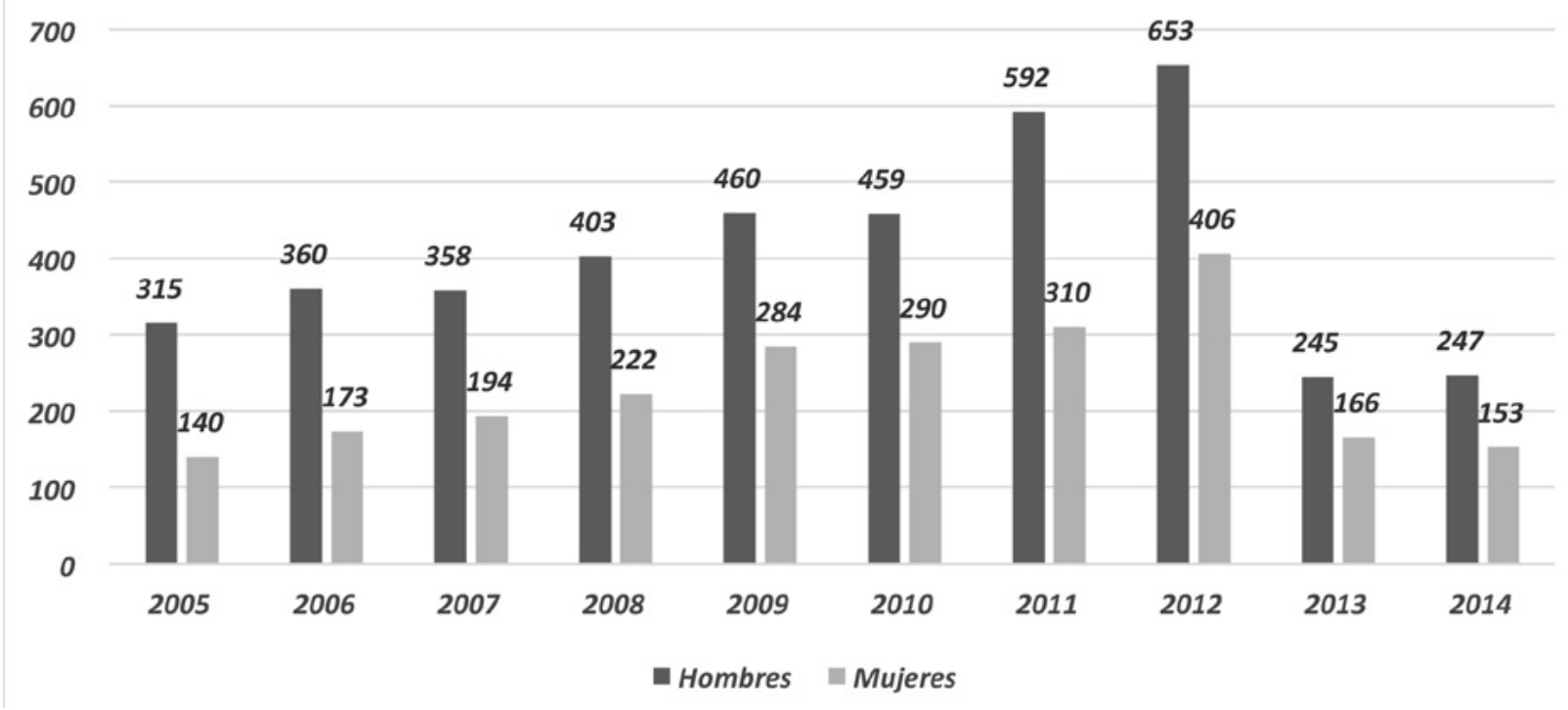


Figura 2. Doctores en el área de ingeniería y tecnología en Colombia según género: 2005-2014. Fuente: OCYT (2016)

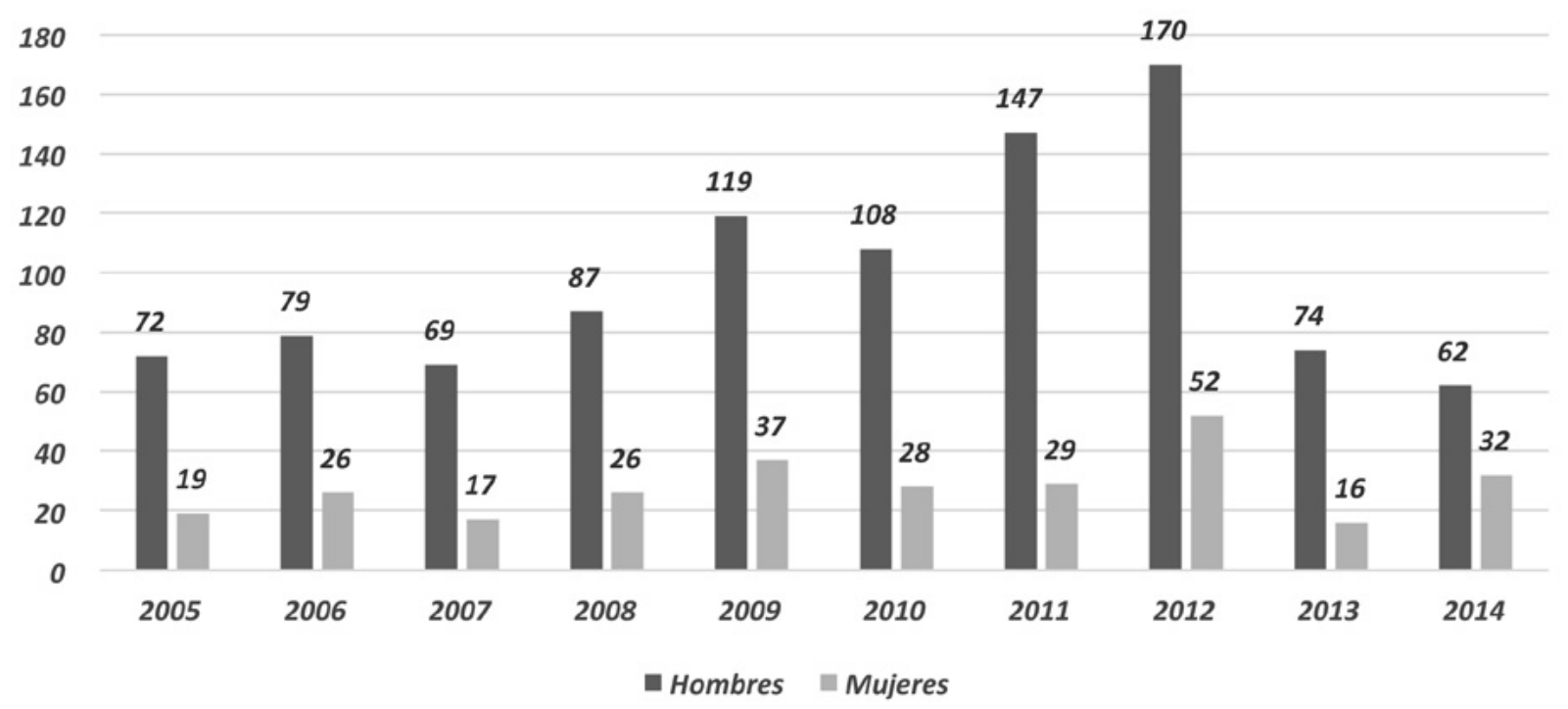

En este contexto, se encuentran las mujeres ingresando, cursando y graduándose de programas de doctorado en ingeniería. Ellas son parte del engranaje de la función de investigación de las universidades y al mismo tiempo del fortalecimiento de las capacidades de investigación e innovación del SNCTI. La Tabla 1 muestra que el porcentaje de mujeres inscritas con respecto al total de inscritos para cursar estudios de doctorado en el año 2004 era del 10,3\%, pero este porcentaje fue del 27,4 \% diez años después. Así mismo, se evidencia un crecimiento en el porcentaje de estudiantes matriculadas: 18,5 \% en 2004 y $28,5 \%$ en 2014 . El porcentaje de mujeres graduadas también creció en el mismo periodo, pasando de $15,4 \%$ a $30 \%$ respectivamente.

A pesar del aumento en la representación de las mujeres en los programas de doctorado en el área de ingeniería en la última década, en Colombia no se han llevado a cabo estudios para conocer, describir y analizar las preferencias de empleo que tienen las estudiantes de doctorado en ingeniería sobre la academia y la industria vistas ambas como los destinos laborales que ellas pueden seleccionar una vez egresen de sus respectivos programas de doctorado (Roach \& Sauermann, 2010; Sauer- mann \& Roach, 2012a). Las investigaciones que se han realizado hasta ahora, sobre las preferencias de empleo, han estado centradas en contrastar éstas con las de los graduados de doctorado y los profesores que laboran en programas de doctorado en ingeniería (Celis, Duque, \& Ramírez, 2012) y comparar dichas preferencias con las que tienen los estudiantes en Estados Unidos en ingeniería y ciencias (Celis \& Duque, 2014).

En efecto, este trabajo puede considerarse como un aporte a la investigación sobre el mercado de los doctores desde la perspectiva de las preferencias de empleo de los estudiantes (Agarwal \& Ohyama, 2013; Cohen, 2010; Stephan, 2012), pero dando un especial énfasis a las preferencias de las estudiantes de doctorado en ingeniería. Por otra parte, los resultados de este trabajo pueden servir para fundamentar estrategias tendientes a aumentar la presencia de las mujeres tanto en el sector académico como en el industrial, pues las preferencias de empleo son un poderoso predictor sobre los destinos laborales de los profesionales con título de doctorado (academia o industria) y de su permanencia en ellos durante el paso del tiempo en el mercado de trabajo (Agarwal \& Ohyama, 2013; Balsmeier \& Pellens, 2015). 
Tabla 1.

Número de inscritos, matriculados y graduados según género en programas de doctorado en el área de ingeniería en Colombia: 2004-2014

\begin{tabular}{|c|c|c|c|c|c|c|c|c|c|}
\hline \multirow{2}{*}{ Año } & \multicolumn{3}{|c|}{ Número de inscritos } & \multicolumn{3}{|c|}{ Número de matriculados } & \multicolumn{3}{|c|}{ Número de graduados } \\
\hline & Hombres & Mujeres & Total & Hombres & Mujeres & Total & Hombres & Mujeres & Total \\
\hline 2004 & 35 & 4 & 39 & 123 & 28 & 151 & 11 & 2 & 13 \\
\hline 2005 & 50 & 14 & 64 & 262 & 69 & 331 & 7 & 1 & 8 \\
\hline 2006 & 74 & 24 & 98 & 319 & 89 & 408 & 14 & 6 & 20 \\
\hline 2007 & 122 & 36 & 158 & 469 & 136 & 605 & 11 & 1 & 12 \\
\hline 2008 & 120 & 33 & 153 & 594 & 183 & 777 & 22 & 11 & 33 \\
\hline 2009 & 175 & 63 & 238 & 692 & 212 & 904 & 34 & 6 & 40 \\
\hline 2010 & 187 & 87 & 274 & 810 & 275 & 1.085 & 33 & 7 & 40 \\
\hline 2011 & 263 & 86 & 349 & 958 & 370 & 1.328 & 58 & 17 & 75 \\
\hline 2012 & 269 & 109 & 378 & 1.093 & 436 & 1.529 & 62 & 23 & 85 \\
\hline 2013 & 308 & 135 & 443 & 1.387 & 570 & 1.957 & 61 & 14 & 75 \\
\hline 2014 & 345 & 130 & 475 & 1.563 & 623 & 2.186 & 62 & 32 & 94 \\
\hline
\end{tabular}

Fuente: MEN-SNIES (2016) y OLE (2016)

De igual manera, los resultados son un sustrato para apoyar los procesos de orientación profesional que son condición básica para apoyar el proceso de selección de un programa de doctorado y una posterior inserción al mercado de trabajo (Sauermann \& Roach, 2012a). El artículo está organizado como sigue. En primer lugar, se ofrece un análisis de estudios que han abordado la situación de las mujeres con doctorado en el mercado de trabajo. Como se evidenciará, la mayoría de los estudios se han concentrado en la academia siendo la industria un destino laboral poco investigado. Posteriormente, se presenta una definición operativa sobre las preferencias de empleo para luego concentrarse en la metodología empleada por este trabajo para conocer las preferencias de empleo de las estudiantes. Paso seguido, se describen las preferencias de empleo de las estudiantes y se contrastan con las de los estudiantes. Finalmente, se hace una discusión de los resultados encontrados y se presentan las conclusiones de este trabajo señalando temas futuros de investigación para seguir profundizando en la comprensión de las preferencias de empleo.

\section{Las mujeres con doctorado en el mercado laboral}

\section{Presencia de las mujeres en la academia}

A pesar de que más mujeres están accediendo a los programas de doctorado y los hombres están menos interesados en optar por una carrera académica posterior a la obtención del título de doctorado (Wolfinger, Mason, \& Goulden, 2009), las mujeres continúan con una participación menor en comparación con la que tienen los hombres en el ámbito universitario (Auriol, Misu, \& Freeman, 2013). Pero el problema no es sólo de una menor participación. Las mujeres que logran acceder a la academia lo hacen en condiciones laborales menos favorables con respecto a las de los hombres. Para el año 2013, por ejemplo, en Europa solamente el $37 \%$ y el $20 \%$ de las mujeres respectivamente ocupaban los Grados B y A que corresponden a los grados más altos del escalafón docente (Evers \& Sieverding, 2015). El porcentaje de mujeres en Grado A fue de 19,8 \% en 2010 mostrando que la 
variación ha sido nula con respecto al año 2013. En Alemania, por su parte, sólo el $18 \%$ de mujeres alcanzaron el rango más alto (full profesor) en 2009 , lo cual contrasta con un incremento del $76 \%$ en la tasa de graduación de mujeres de doctorado en los últimos 17 años (Evers \& Sieverding, 2015).

Por otro lado, en Estados Unidos para el año 2005 solamente el $10 \%$ de los profesores titulares o que estaban en proceso de alcanzar esta categoría eran mujeres (Chubin, May, \& Babco, 2005). Tradicionalmente, la educación superior ha sido un sector con una participación mayoritariamente masculina. En algunos países como Austria se han creado programas dirigidos a aumentar la presencia de las mujeres en la academia (Fritsch, 2016). Ahora bien, los análisis sobre las mujeres con doctorado en el ámbito universitario concluyen que es más probable que ellas se desempeñen en áreas administrativas antes que en posiciones académicas como suele suceder con los hombres (Conti \& Visentin, 2015b). Adicionalmente, tienen un $25 \%$ más de probabilidad de tener un empleo que no sea de docencia en una universidad que los hombres (Wolfinger et al., 2009). Se ha encontrado en el $43 \%$ de los casos que es más probable que las mujeres tengan el rol de docente adjunto u otros cargos que no son de carrera en relación con los cargos que ostentan los hombres (Wolfinger et al., 2009).

Sin embargo, es más factible que las mujeres trabajen en universidades que en compañías de investigación y desarrollo (l+D) (Conti \& Visentin, 2015b). Auriol et al. (2013) encuentra que el mercado de los doctores en 19 países estudiados en todos menos en 5 , la proporción de contratos de tiempo parcial es mayor para las mujeres comparado con los de los hombres. En este análisis se identifica también que existen formas de contratación diferentes a la carrera docente, como contratos de medio tiempo o de docentes adjuntos conllevan ingresos salariales más bajos: los docentes adjuntos reciben un $26 \%$ menos de ingresos que los profesores de carrera (Wolfinger et al., 2009) y dado que es más probable que las mujeres tengan estas formas más desfavorables de contratación, sus ingresos resultan ser en general inferiores a los de los hombres.
Otros factores como el estado civil y tener hijos afectan negativamente las posibilidades de que las mujeres puedan ingresar a la carrera docente. En contraposición, estos factores no tienen la misma incidencia en los hombres. Cuando las mujeres tienen hijos es $20 \%$ menos probable que lleguen a convertirse en profesoras titulares o de carrera (Wolfinger et al., 2009).

Por otro lado, en cuanto a la empleabilidad de las mujeres, mientras que las probabilidades de ser parte de la academia son menores para las mujeres, como se ha venido exponiendo, las de quedar sin empleo son mayores para ellas, especialmente cuando tienen hijos menores de 6 años o están en edad preescolar. En 13 de los 19 países del estudio realizado por Auriol et al. (2013), además, se evidencia una tasa mayor de desempleo en las mujeres con doctorado comparativamente a la de los hombres empero, la tasa de desempleo es en general menor, tanto para hombres como para mujeres con doctorado, al contrastarla con personas con niveles educativos inferiores (Auriol et al., 2013). En relación con la empleabilidad, Enders (2002) indica que dos años después de terminar el doctorado, la tasa de desempleo para las mujeres es del $4 \%$ mientras que para los hombres es tan solo del $2 \%$. Adicionalmente, un $6 \%$ de las mujeres graduadas de doctorado deja el mercado laboral para dedicarse al cuidado de su familia, una situación que casi no se presenta con los hombres. En Colombia, aunque no se evidencia diferencias significativas de género en las tasas de empleabilidad, se encuentra que las mujeres con doctorado perciben menores ingresos frente a los hombres (Celis \& Duque, 2014). Así, las mujeres han pasado de recibir el equivalente al 93,8 \% de los ingresos de los hombres en 2007, al 87 \% en 2014 (Ver Tabla 2).

En cuanto a la empleabilidad por área de conocimiento, el estudio de Auriol et al. (2013) reporta que en las Ciencias Naturales e Ingenierías hay mayor nivel de empleabilidad en investigación en comparación con el de las Ciencias Sociales. En el área de la Salud, los graduados reciben mayores ingresos en comparación con otras áreas como Ciencias Sociales e Ingenierías, y las tasas de desempleo son más altas en Humanidades y menores en Ciencias e Ingenierías. 
Tabla 2.

Ingresos y tasas de cotizantes de doctores por género. 2007-2014

\begin{tabular}{ccccc}
\hline \multirow{2}{*}{ Año } & \multicolumn{2}{c}{ Hombres } & \multicolumn{2}{c}{ Mujeres } \\
\cline { 2 - 5 } & Ingresos (\$) & Tasa empleabilidad (\%) & Ingresos (\$) & Tasa empleabilidad (\%) \\
\hline 2007 & 4.755 .432 & 91,6 & 4.458 .682 & 95,3 \\
\hline 2008 & 5.140 .062 & 93,6 & 4.559 .154 & 96,7 \\
\hline 2009 & 5.563 .452 & 94,1 & 4.848 .098 & 94,4 \\
\hline 2010 & 5.726 .732 & 93,8 & 4.969 .678 & 93,6 \\
\hline 2011 & 5.936 .666 & 93,1 & 5.175 .155 & 91,5 \\
\hline 2012 & 6.401 .817 & 93,8 & 5.530 .612 & 91,3 \\
\hline 2013 & 6.563 .528 & 94,4 & 5.686 .734 & 90,4 \\
\hline 2014 & 6.730 .473 & 94 & 5.856 .272 & 91,5 \\
\hline
\end{tabular}

Fuente: OLE (2016).

En un estudio sobre los doctores en el área de la biomedicina, se concluye que los ingresos salariales de las mujeres son menores que los de los hombres con doctorado y que los hombres muestran mayor interés por empleos de investigación en universidades mientras que las mujeres, que hacen parte de minorías, presentan mayor inclinación por empleos que no sean de investigación (Gibbs, McGready, Bennett, \& Griffin, 2014).

Por otro lado, con respecto al impacto de los factores familiares en las mujeres se encuentra que las características familiares son un factor de análisis frente a la empleabilidad de las mujeres graduadas de programas de doctorado y de su tipo de vinculación con las universidades y con el sector productivo (Evers \& Sieverding, 2015; Wolfinger et al., 2009). Estas características se refieren, por un lado, a la conformación de una familia nuclear (Enders, 2002) y, por otro, a la familia de origen y la relación de ésta con el mundo académico (Herrera, Baca, Aguilar, \& Nieto, 2013). De esta manera, un aspecto que afecta el cambio de las metas de las mujeres en relación con el desarrollo de una carrera académica es tener hijos (41\%) o planear tenerlos ( $28 \%$ ); mientras que en hombres la incidencia es menor, $20 \%$ tenerlos y $17 \%$ planear tenerlos respectivamente (Evers \& Sieverding, 2015). Así pues, las características fa- miliares, además de afectar el curso de la carrera académica, influyen en el éxito en el desempeño, especialmente en mujeres. De acuerdo con Enders (2002) mientras que los hombres con esposa e hijos tienden a ser profesionales más exitosos que los solteros, las mujeres casadas con hijos son menos exitosas que mujeres solteras. En ese mismo sentido, aunque las discontinuidades de la vida profesional, como las pausas por paternidad, tienen un impacto negativo sobre los logros de la carrera, tanto en hombres como en mujeres, afectan especialmente a las mujeres (Enders, 2002; Evers \& Sieverding, 2015).

Continuando con el análisis del éxito laboral, a partir de una clasificación entre trabajos de alto nivel y otros de bajo nivel, con respecto a la posición jerárquica, ya sea dentro o fuera de la academia, solo el $31 \%$ tiene un cargo de alto nivel, mientras que en el caso de la muestra de los hombres el 51 tiene este tipo de empleos (Bornmann \& Enders, 2004). En este mismo sentido Clancy, Nelson, Rutherford, y Hinde (2014) encuentran entre sus encuestados que, aunque reportan haber trabajado en 3,2 lugares diferentes, solamente en 1,2 de estos han estado liderados por una mujer.

En cuanto al clima laboral y las experiencias de acoso y agresión sexual, se encuentra que el clima 
laboral es visto como un factor determinante a la hora de comprender la participación que tienen las mujeres en la academia, especialmente aquellas variables que tienen relación con el género y las experiencias de acoso y agresión sexual. Clancy et al. (2014) aplicaron una encuesta a 666 personas, de las cuales el 77,5 \% eran mujeres, y algunas ellas eran estudiantes de pregrado y posgrado, investigadores en estancias postdoctorales, profesores asistentes, de carrera y jubilados, empleados en investigación y otras no tenían relación con el mundo universitario. La encuesta indagaba por situaciones para las cuales los participantes debían seleccionar la frecuencia con que situaciones de acoso y agresión sexual se presentaban en sus espacios laborales. Los hallazgos mostraron marcadas diferencias de género en las respuesta. Mientras los reportes de los hombres estaban más orientados a calificar la frecuencia de las situaciones como nunca ocurre, la frecuencia para el caso de las mujeres fue ocurre frecuentemente. Del total de los encuestados, el 72,4 \% reportaron haber observado directamente o haber recibido información sobre colegas o investigadores que hacían comentarios inapropiados o sexuales en los espacios de trabajo. En cuanto a las experiencias de acoso sexual ${ }^{1}$, el $64 \%$ de los encuestados reportaron haber sufrido este tipo de acoso. Por su parte, el 21,7 \% manifestó haber tenido experiencias directas de agresión sexual reflejadas en situaciones como contacto físico indeseado o relaciones sexuales no consentidas (Clancy et al., 2014). Las principales víctimas del acoso y la agresión sexual fueron los estudiantes, los post-doctores y los empleados. Más del $90 \%$ de las mujeres ocupaban alguno de estos roles cuando fueron víctimas y en el caso de los hombres más del $70 \%$. De otro lado, se encontró que el género puede considerarse un predictor para ser víctima de acoso sexual, siendo 3,5 más probable que las mujeres tengan este tipo de experiencias (Clancy et al., 2014).

Con lo anterior, el análisis de la relación entre víctimas y perpetradores permite concluir que para el caso de los hombres los perpetradores suelen ser sus pares. En contrapartida, los perpetradores de las mujeres son sus superiores jerárquicos,

1 Entendidas como comentarios inapropiados o belleza física, diferencias cognitivas entre sexos y otra clase de bromas mostrando así una relación de poder. Las experiencias de acoso y agresión sexual en los espacios académicos pueden considerarse factores de riesgo para el adecuado desarrollo profesional, especialmente en las mujeres quienes se ven más afectadas tanto por el acoso sexual como por las agresiones de este tipo. La creación de códigos de conducta y las políticas frente al acoso y agresión sexual en el interior de los espacios académicos laborales pueden convertirse en herramientas para prevenir estas situaciones (Clancy et al., 2014).

Adicional a esto, los ingresos son otro aspecto clave de análisis frente a la empleabilidad según el género. La desigualdad en los ingresos entre hombres y mujeres se presenta tanto en el sector productivo como en el académico (Bornmann \& Enders, 2004; Enders, 2002). Aunque esta diferencia está presente en todas las disciplinas, es más marcada en algunas de ellas. Por ejemplo, en economía y administración los hombres ganan un $46 \%$ más que las mujeres mientras que en ciencias sociales la diferencia a favor de los hombres es de $20 \%$ (Bornmann \& Enders, 2004). Es preciso advertir que, aunque hay una idea generalizada sobre la desigualdad en ingresos entre hombres y mujeres, algunos autores consideran que falta una medición y comparabilidad más rigurosa al respecto (Auriol et al., 2013). En este sentido, a pesar de la media de ingresos masculina es mayor a la femenina, no existe una comparación metodológicamente robusta entre los ingresos que perciben las mujeres frente al de los hombres debido a que no se tienen en cuenta aspectos como la experiencia, la antigüedad o el tipo de contratación (Auriol et al., 2013). Finalmente, vale la pena destacar que para comprender las causas de la baja representación de las mujeres en la ciencia es necesario tener en cuenta aspectos propios de elección personal (Ceci, Ginther, Kahn, \& Williams, 2014; Evers \& Sieverding, 2015). Por ejemplo, inicialmente hombres y mujeres tienen la misma inclinación por cierta cantidad de horas de trabajo, pero con la edad, aproximadamente a los 30 años, las mujeres prefieren trabajar menos horas y en ese sentido toman decisiones sobre su empleo (Ceci et al., 2014). Estas elecciones personales pueden estar centradas en el trabajo, en 
la familia o en equilibrar ambos (Evers \& Sieverding, 2015) y resultan de primer orden para comprender la representación femenina dentro de la ciencia y el rol de las mujeres en los campos laborales en que se desempeñan cuando egresan del doctorado.

\section{Qué son las preferencias de empleo}

Este trabajo asume las preferencias de empleo como el grado de interés que tienen las estudiantes de doctorado por un conjunto de atributos laborales que se encuentran en un empleo bien sea en la academia o en la industria. La selección de un determinado empleo es hecha por las estudiantes de acuerdo con el grado de interés que tienen sobre ciertos atributos laborales asociados al empleo deseado o mayor cercanía con su proyecto profesional futuro (Celis \& Duque, 2014). Varios estudios clasifican los atributos laborales entre motivos intrínsecos y extrínsecos (Roach \& Sauermann, 2010; Sauermann, 2005, 2008; Sauermann \& Roach, 2014). Los primeros están relacionados con la satisfacción que obtienen los profesionales con doctorado al realizar aquellas tareas que son consustanciales al mismo trabajo mientras los segundos están relacionados con las condiciones objetivas que ofrece un empleo (Cohen \& Sauermann, 2007). La literatura ha venido clasificando los siguientes atributos como parte de los motivos intrínsecos: 1) retos intelectuales; 2) libertad para escoger proyectos de investigación; 3) nivel de responsabilidad en el trabajo; 4) posibilidad de colaborar con otras instituciones/organizaciones; 5) opciones de ganar reconocimiento de los pares; 6) posibilidades de publicar resultados de investigación. Por su parte, atributos como: 1) salario y beneficios; 2) estabilidad (aboral; 3) disponibilidad de fondos y recursos; y 4) disponibilidad de tecnologías y equipamiento de punta están relacionados con los motivos extrínsecos.

En las investigaciones sobre las preferencias de empleo se encuentra que los estudiantes que exhiben un alto interés por los atributos laborales relacionados con los motivos intrínsecos tienen mayor inclinación por la academia, mientras que aquellos con alta valoración por los motivos extrínsecos suelen valorar más la industria (Roach
\& Sauermann, 2010, 2012; Sauermann \& Roach, 2012b). Los estudiantes con alta preferencia por los motivos intrínsecos se dice que tienen gusto por la ciencias. En cambio, aquellos con preferencia por los motivos extrínsecos tiene gusto por la comercialización (Sauermann \& Roach, 2012b). Algunos estudios señalan que una fuerte inclinación por parte de los estudiantes por publicar explica en un alto grado de interés por ingresar a la academia (Fox \& Stephan, 2001; Mangematin, 2000; Roach \& Sauermann, 2010; Sauermann \& Roach, 2014) y en la permanencia misma de los doctores en la carrera académica pasados varios años de experiencia (Balsmeier \& Pellens, 2015).

En cambio, disponer de tecnología y equipamiento de punta y salarios altos son atributos que son altamente apreciados por aquellos estudiantes que desean trabajar en la industria (Celis et al., 2012; Roach \& Sauermann, 2010). Estos hallazgos deben ser interpretados con precaución. Estudios sobre los doctores que laboran tanto en la academia como en la industria concluyen que los aquellos que están en la industria también aprecian las posibilidades de publicar y de hecho cuentan con un record de publicaciones. Esto también sucede en la aprecian igualmente las posibilidades de patentar y de hecho tienen patentes registradas a su nombre (Roach \& Sauermann, 2012; Sauermann \& Roach, 2012b, 2014).

\section{Método}

En este estudio se aplicó una encuesta virtual a un grupo de estudiantes que estaban cursando un programa de doctorado en ingeniería en una universidad en Colombia con el fin de identificar sus preferencias de empleo. Las preguntas se tomaron de trabajos de investigación similares sobre preferencias de empleo con el propósito de garantizar un nivel de validez apropiado. La encuesta se tuvo disponible durante un periodo aproximado de nueve semanas, lapso en el que se enviaron tres recordatorios a los correos electrónicos de los estudiantes con el fin de garantizar una alta tasa de respuesta. Las preguntas fueron cerradas y para medir las preferencias de empleo se utilizó escala de Likert. El procesamiento estadístico, cálculo de las medias y de las frecuencias, se 
realizó a través de las herramientas de la plataforma en línea en la que se dispuso la encuesta. De un total de 105 estudiantes, 63 estudiantes efectivamente respondieron la encuesta. De ellos 12 $(19,05 \%)$ eran mujeres.

La encuesta abordó temas relacionados con el interés que tienen los estudiantes por diferentes posiciones laborales, un grupo de atributos laborales y unas actividades realizadas en un empleo. De acuerdo con la definición sobre las preferencias de empleo, este estudio definió las cuatro variables para identificar las preferencias de empleo de las estudiantes: 1) nivel de interés por diferentes posiciones laborales 2) nivel de importancia por varios atributos laborales 3 ) nivel de interés por varias actividades y 4) gusto por la ciencia y gusto por la comercialización. A continuación, se presenta la operacionalización de las variables:

Nivel de interés por diferentes posiciones laborales. A los estudiantes se les preguntó qué tan interesante eran para ellos ciertas posiciones laborales una vez concluyeran su formación doctoral usando. Para este fin, se empleó una escala de 1 a 5 donde 1 significaba nada interesante y 5 extremadamente interesante. Estas posiciones fueron organizadas en tres grupos: posiciones laborales académicas, posiciones laborales que no son académicas y posiciones laborales no basadas en la investigación (Gibbs et al., 2014). Las posiciones laborales académicas fueron post-doctorado en una universidad y profesor con énfasis en investigación y en enseñanza. Las posiciones laborales que no eran académicas fueron: investigador en una centro de investigación público o privado y en una empresa. Las posiciones laborales no basadas en la investigación fueron docente en un colegio, consultor en ingeniería, gerente en una empresa y servidor público.

Nivel de importancia por varios atributos laborales. A los estudiantes se les preguntó sobre el nivel de importancia que otorgaban a los siguientes atributos laborales: 1) retos intelectuales; 2) libertad para escoger proyectos; 3) nivel de responsabilidad en el trabajo; 4) posibilidad de colaborar con otras instituciones/organizaciones; 5) opciones de ganar reconocimiento de los pares;
6) publicar resultados de investigación; 7) salario y beneficios; 8) estabilidad laboral; 9) disponibilidad de fondos y recursos; y 10) disponibilidad de tecnologías y equipamiento de punta. La pregunta se hizo con una escala de 1 a 5 en donde 1 era nada importante y 5 extremadamente importante.

Nivel de interés por varias actividades. A los estudiantes se les pidió valorar qué tan interesante eran las siguientes actividades en el puesto de trabajo de su preferencia: 1) investigación básica; 2) investigación aplicada; 3) desarrollo; 4) programación; 5) diseño; 6) administración; y 7) enseñanza (NSF, 2013). La pregunta se hizo con una escala de 1 a 5 ( 1 = nada interesante; 5 = extremadamente interesante).

Gusto por la ciencia (GCi) y gusto por la comercialización (GCo). Para determinar aquellos estudiantes con $\mathrm{GCi}$ se estableció que serían aquellos que consideraban profesor universitario con énfasis en investigación como la posición laboral más importante y la investigación básica como la actividad más interesante. Además, tenían un alto nivel de interés en los atributos asociados a los motivos intrínsecos. En contrapartida, aquellos estudiantes con GCo serían los que mostraron mayor interés por investigador en una empresa, gerente de una empresa o consultor en ingeniería como las posiciones laborales más importantes. Adicionalmente, serían aquellos que afirmaron tener un alto nivel de interés en la investigación aplicada y los atributos relacionados con los motivos extrínsecos.

\section{Resultados}

Los resultados obtenidos se abordarán presentando de manera comparativa las respuestas de hombres y mujeres. En primer lugar, se presentará el nivel de interés de los encuestados por diferentes posiciones laborales. Posteriormente, el nivel de importancia asignada a ciertos atributos laborales y luego al nivel de interés por determinadas actividades. Por último, un análisis a partir de los resultados en las diferentes variables en relación con el gusto que tienen las encuestadas por la comercialización en contraposición con el gusto por la ciencia. 
En cuanto al nivel de interés por diferentes posiciones laborales, las mujeres mostraron mayor interés por las posiciones de investigadora en un centro de investigación privado y profesora con énfasis en investigación (ver Figura 3). Es importante señalar que se asigna el mismo nivel de interés para ambas posiciones, esto fue de 4,33. En el caso de los hombres, ser profesor con énfasis en investigación fue la posición de mayor interés $(4,31)$. Seguida de la posición de investigador en un centro de investigación privado $(4,08)$. Aunque son las mismas posiciones que interesan mujeres y hombres, lo cierto lo que se evidenció es que las mujeres tienen un mayor nivel de interés en estas dos posiciones comparado con el que tienen los hombres.

Por otra parte, las posiciones laborales en las que las mujeres tienen menos interés fueron gerente de una empresa y docente en un colegio. En estas posiciones las mujeres exhibieron más desinterés, para ambos casos fue de 1,92. Los hombres presentaron poco interés por estas posiciones, a saber, gerente de una empresa $(3,14)$ y docente en un colegio $(1,51)$. Llama la atención que las mujeres tengan un mayor desinterés que los hombres en la posición gerente en una empresa.

Figura 3. Nivel de interés por diferentes posiciones laborales según género

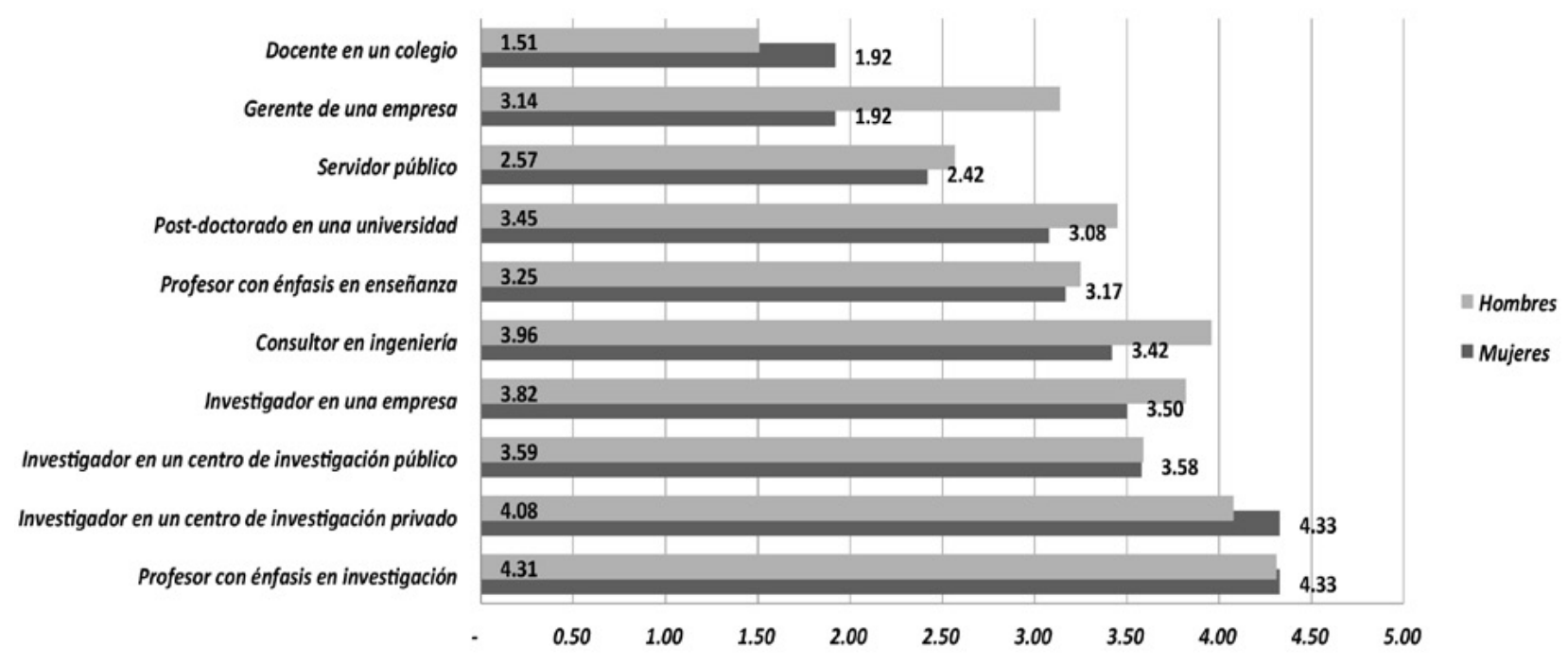

Estos resultados ponen de manifiesto el interés de las mujeres por desempeñarse en el ámbito académico y en aquellos espacios en los cuales puedan realizar investigación. En este sentido es pertinente resaltar que en general, de acuerdo con la literatura, las condiciones de empleo son menos favorables para las mujeres (Auriol et al., 2013; Wolfinger et al., 2009) y los espacios dispuestos para ellas no son equiparables con los de los hombres (Auriol et al., 2013; Evers \& Siever- ding, 2015; Fritsch, 2016), especialmente en el área de la investigación en la que es menos probable que las mujeres participen según lo reportado por Conti and Visentin (2015a).

En cuanto al nivel de importancia otorgada a ciertos atributos laborales, se encuentra que los dos atributos que más valoraron las estudiantes fueron la disponibilidad de fondos y recursos y la posibilidad de colaborar con otras instituciones/ 
organizaciones. Las mujeres otorgaron a estos dos atributos el mismo nivel de importancia: 4,50 (ver Figura 4). En cambio, retos intelectuales fue el atributo más importante para los hombres, esto fue de 4,67. Llama la atención esa diferencia entre hombres y mujeres con respecto a los atributos que ambos grupos consideran como los más importantes. Mujeres y hombres coinciden en afirmar que el atributo con menor importancia fue la opción de ganar reconocimiento de los pares, esto fue 2,58 y 3,35 respectivamente.
Mientras las mujeres asignaron un mayor nivel de importancia a la posibilidad de publicar resultados $(4,25)$, los hombres valoraron más el salario y los beneficios $(4,18)$. Los hombres valoraron más la disponibilidad de tecnologías y equipamiento de punta $(4,06)$ que las mujeres y éstas dieron más peso a la estabilidad laboral $(4,25)$ que los hombres. Fueron similares las valoraciones que ambos grupos dieron a los atributos libertad para escoger proyectos y nivel de responsabilidad en el trabajo.

Figura 4. Nivel de importancia otorgada a ciertos atributos laborales según género.

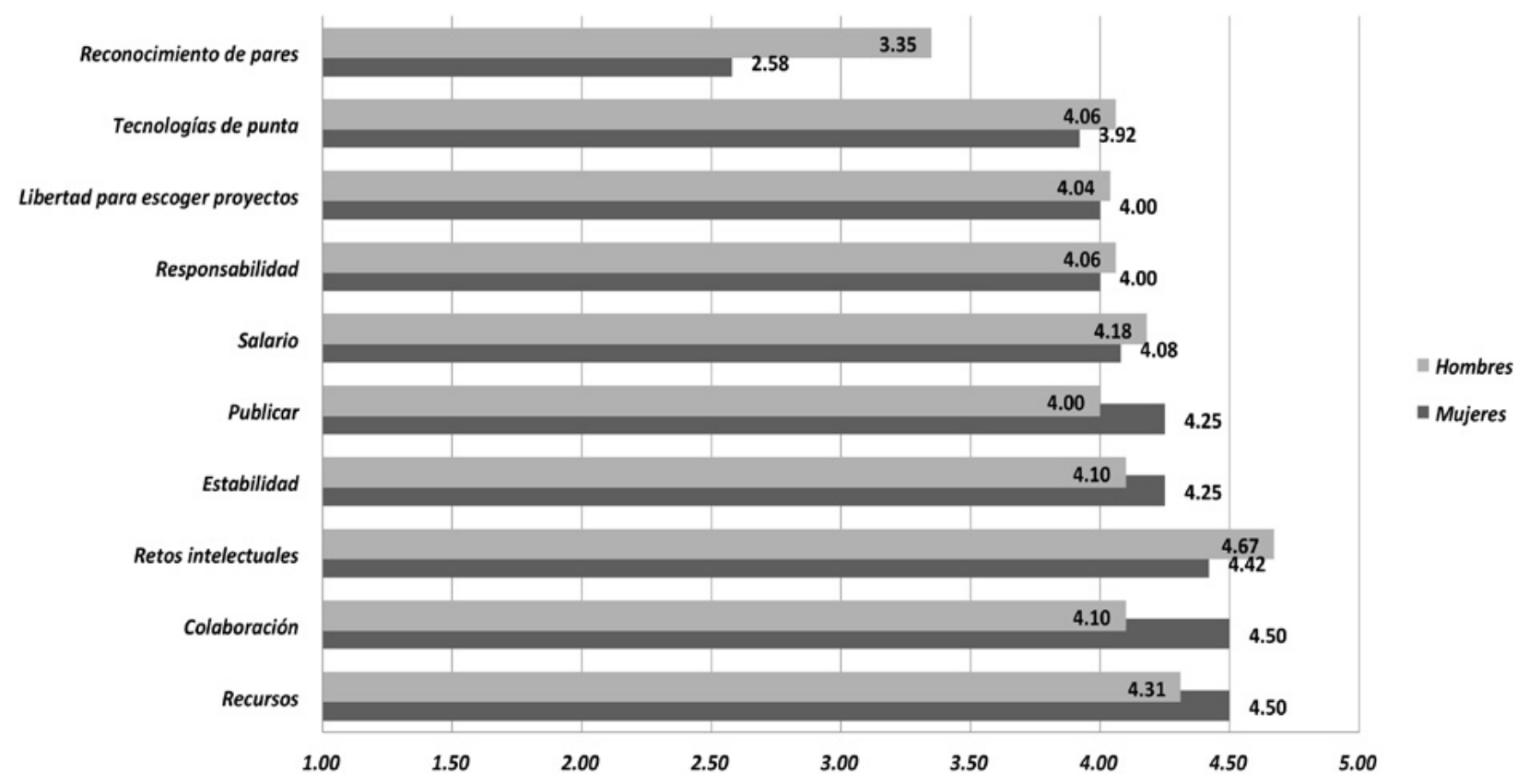

Al analizar el nivel de interés por actividades en un empleo de su preferencia, tanto los hombres como las mujeres presentaron el mismo nivel de interés por la investigación aplicada, esto fue 4,37 y 4,25 respectivamente. De igual manera, consideraron la programación la actividad menos atractiva, aunque las mujeres la percibieron menos interesante $(3,00)$ que los hombres $(3,55)$ (ver Figura 5$)$. Mientras que las mujeres encontraron más interesante la enseñanza $(3,83)$, la investigación básica fue más interesante para los hombres $(3,90)$.
El gusto por la ciencia y la comercialización en las mujeres obtuvo respuestas por parte de las mujeres que permitieron identificar una tendencia, aunque no un único perfil, en relación con el gusto por la ciencia en contraposición con el gusto por la comercialización. Esto se evidencia en un interés mayor que el de los hombres por posiciones relacionadas con la enseñanza, no sólo la docencia con énfasis en investigación sino incluso la docencia en colegios que, si bien no fue una de sus preferencias principales, fue mejor puntuada en su caso que en el de los hombres. En contraposición, 
las posiciones de menor interés para las mujeres fueron las de gerente de empresa y consultor de una empresa, ambas posiciones relacionadas con un perfil de gusto por la comercialización.

La lectura de esta tendencia se encontró en las respuestas de las mujeres en cuanto a los atributos de los empleos. Si bien puntuaron alto en la disponibilidad de recursos, atributo que fue asociado con el gusto por la comercialización, en el mismo lugar estuvo el interés por la colaboración y un poco menos por los retos intelectuales, ambos, atributos asociados con el gusto por la ciencia.

De los cinco atributos de mayor interés para las mujeres, tres estuvieron asociados con el gusto por la ciencia, esto fue, colaboración, retos intelectuales y publicación. Sin embargo, en cuanto al interés por ciertas actividades asociadas con el empleo, el primer lugar lo ocupó la investigación aplicada, más relacionada con el gusto por la comercialización. No obstante, los dos siguientes lugares correspondieron a la enseñanza y a la investigación básica; atributos relacionados con el gusto por la ciencia.

Estos resultados sugieren la pertinencia de profundizar en los factores que determinan las preferencias de empleo para el caso específico de las mujeres que se gradúan de programas de doctorado del campo de la ingeniería en futuras investigaciones.

Figura 5. Nivel de interés por actividades en un empleo de su preferencia según género.

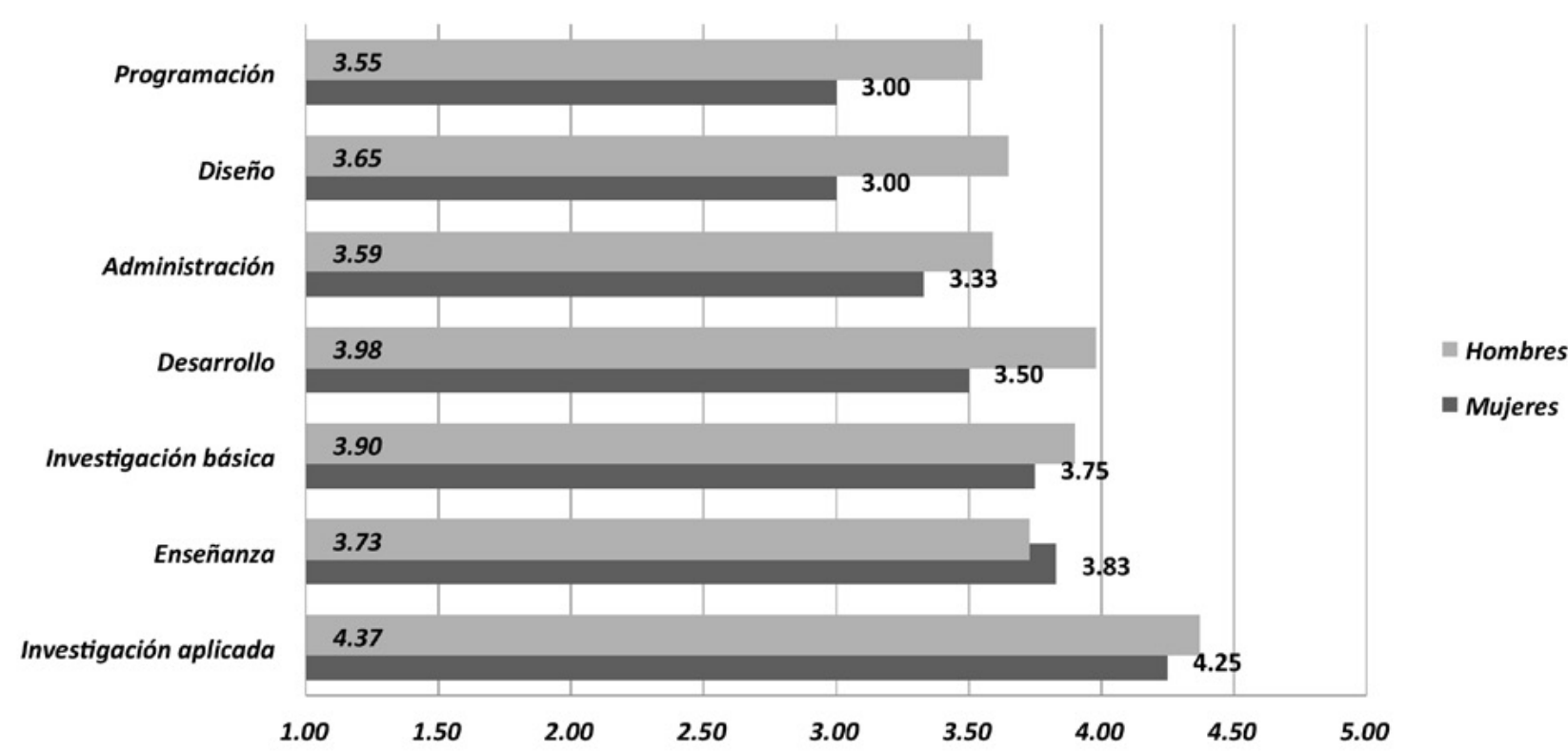

\section{Discusión y conclusiones}

Este trabajo puede ser considerado un aporte a los estudios de género en particular y del mercado de trabajo de los doctores en Colombia en general. Es indiscutible que las preferencias de empleo de las mujeres difieren de las de los hombres. Así, mientras las mujeres presentaron una mayor in- clinación por los atributos laborales que caracterizan a la academia, los hombres evidencian una mayor por los relacionados con la industria y se mostraron menos interesados en optar por una carrera académica, factor que favoreció el ingreso de las mujeres a la academia (Wolfinger et al., 2009). 
Este hallazgo puede soportarse en las diferencias encontradas en los resultados que muestran particularidades según el género, a partir de los cuales un análisis global permite afirmar que las mujeres presentaron un mayor gusto por la ciencia, la docencia y la investigación. Las preferencias de empleo de las mujeres que cursan programas de doctorado en ingeniería no han sido investigadas y mucho menos se han contrastado con las de los hombres. Entender las preferencias de empleo de las mujeres es un insumo básico para concebir y diseñar programas que contribuyan a su ubicación laboral una vez concluyan su formaciónEn varios países del mundo se han generado acciones afirmativas sobre la inequidad de género en los contextos educativos buscando transformarla (Wolfinger et al., 2009). De la misma manera, existen programas con financiación pública no sólo para estimular a más mujeres a cursar estudios de doctorado en Ingeniería y Ciencias, sino también a ubicarlas en carreras académicas encaminadas a que ocupen las posiciones más altas dentro del escalafón docente (Fritsch, 2016).

Poder identificar las preferencias de empelo de las mujeres y su relación con las condiciones que ofrecen los empleos, ya sean en la academia o en la industria, permitirá que las estrategias para fomentar la presencia de las mujeres con doctorado en el mundo laboral se encaminen a impactar en aquellas variables que actualmente influyen en su baja representación.

Dentro de estas variables se encuentra la constitución de familia, que para el caso de las mujeres se puede convertir en la barrera para iniciar o culminar estudios de doctorado $\mathrm{y}$, posteriormente, para permanecer en el campo laboral sobre todo el de la academia. Es así, que las mujeres parecen verse obligadas a elegir entre la familia y la profesión, lo que no ocurre en el caso de los hombres (Enders, 2002; Evers \& Sieverding, 2015).

Otras variables de marcada importancia fueron el clima laboral, el acoso y la agresión sexual dentro de los contextos laborales, que afectan principalmente a las mujeres, quienes son víctimas especialmente de personas como jefes o superiores afectando así su desempeño profesional (Clancy et al., 2014). También es preponderante tener en cuenta las condiciones de contratación e ingresos que presentan una tendencia desfavorable para las mujeres.

Por otro lado, en Colombia se han empezado a realizar estudios sobre el mercado de trabajo de los doctores desde la perspectiva de las preferencias de empleo de los estudiantes de doctorado (Celis \& Duque, 2014; Celis et al., 2012). Estos estudios muestran que efectivamente las preferencias de empleo tienen una incidencia a la hora de optar por un empleo específico. También señalan que los estudiantes de doctorado, por lo menos en el área de ingeniería, tienen una fuerte inclinación por la academia: tan solo 2 estudiantes de 10 mencionaron tener interés en trabajar en la industria (Celis et al., 2012). De ahí la importancia de introducir los programas de doctorado para la industria que, tal como muestra la evidencia, impactan las preferencias de empleo de los estudiantes hacia la industria como destino laboral (Celis \& Acosta, 2016; Celis \& Duque, 2013).

Ahora bien, es importante que la investigación futura sobre el mercado de trabajo de los profesionales con doctorado incluya con mayor determinación las preferencias de empleo de las mujeres, de tal manera que se puedan conocer los atributos laborales que tienen más peso cuando ellas escogen un empleo en la academia o la industria y si dichos atributos cambian a lo largo del tiempo; sobre todo después de que las mujeres han pasado cierto tiempo en un empleo. Con el fin de tener una comprensión más amplia de las preferencias de empleo que afectan las decisiones de las mujeres a la hora de optar por un empleo, en la academia o en la industria en Colombia, es fundamental hacer estudios que involucren más universidades que ofertan programas de doctorado en el área de ingeniería.

De igual manera, hacer estudios que también consideren las estudiantes de Ciencias - un área en la cual las estudiantes son proclives a trabajar posteriormente en la industria (Roach \& Sauermann, 2010; Sauermann \& Roach, 2012a)- para contrastar sus preferencias de empleo con la de las estudiantes de ingeniería. 


\section{Referencias}

Acosta, O., \& Celis, J. (2014). The emergence of doctoral programmes in the Colombian higher education system: Trends and challenges. Prospects, 44(3), 463-481. doi:10.1007/s11125014-9310-5

Agarwal, R., \& Ohyama, A. (2013). Industry or Academia, Basic or Applied? Career Choices and Earnings Trajectories of Scientists. Management Science, 59(4), 950-970.

Altbach, P. G., \& Jamil, S. (2011). The Road to Academic Excellence. The Making of World-Class Research Universities. Washington: The World Bank.

Auriol, L., Misu, M., \& Freeman, R. (2013). Careers of Doctorate Holders: Analysis of Labour Market and Mobility Indicators. Paris: OECD Publishing.

Balsmeier, B., \& Pellens, M. (2015). How much does it cost to be a scientist? The Journal of Technology Transfer, 1-37. doi:10.1007/s10961-0149388-1

Bornmann, L., \& Enders, J. (2004). Social origin and gender of doctoral degree holders. Impact of particularistic attributes in access to and in later career attainment after achieving the doctoral degree in Germany. Scientometrics, 61(1), 19-41.

Ceci, S. J., Ginther, D. K., Kahn, S., \& Williams, W. M. (2014). Women in Academic Science: A Changing Landscape. Psychological Science in the Public Interest, 15(3), 75-141. doi:10.1177/1529100614541236

Celis, J., \& Acosta, O. (2016). Industrial Ph.D. Programs for the Strengthening of the Industry's Production of Innovation in Colombia. Revista Innovar, 26(62), 129-146.

Celis, J., Camacho, A., Arenas, A., \& Duque, M. (2014). Dime cómo enseñas y te diré que aprenden los estudiantes. Los aprendizajes y las prácticas de aula en algunas Facultades de
Ingeniería en Colombia. Bogotá: ACOFI, ICFES, UniAndes, UniNorte.

Celis, J., \& Duque, M. (2013). Programas de doctorado en ingeniería para la innovación: una propuesta de política pública para el sector industrial en Colombia. Revista Colombiana de Sociología, 36(2), 163-181.

Celis, J., \& Duque, M. (2014). Estudio comparado sobre preferencias profesionales de doctorados en ingeniería. Colombia-Estados Unidos. Educación y Educadores, 17(2), 306-320.

Celis, J., Duque, M., \& Ramírez, C. (2012). Doctorados en ingeniería para promover la innovación: una propuesta para acrecentar la competitividad empresarial basada en la inserción de doctores en ingeniería en Colombia. Bogotá: ACOFI \& UniAndes.

Chubin, D. E., May, G. S., \& Babco, E. L. (2005). Diversifying the Engineering Workforce. Journal of Engineering Education, 94(1), 73-86. doi:10.1002/j.2168-9830.2005.tb00830.x

Clancy, K. B. H., Nelson, R. G., Rutherford, J. N., \& Hinde, K. (2014). Survey of Academic Field Experiences (SAFE): Trainees Report Harassment and Assault. PLoS ONE, 9(7), e102172. doi:10. 1371/journal.pone.0102172

Cohen, W. (2010). Chapter 4 - Fifty Years of Empirical Studies of Innovative Activity and Performance. In H. H. Bronwyn \& R. Nathan (Eds.), Handbook of the Economics of Innovation (Vol. 1, pp. 129-213). UK: North-Holland.

Cohen, W., \& Sauermann, H. (2007). Schumpeter's Prophecy and Individual Incentives as a Driver of Innovation In F. Malerba \& S. Brusoni (Eds.), Perspectives on Innovation (pp. 73-104). UK: Cambridge University Press.

Colmenares, J., \& Celis, J. (2011). El papel de la universidad en la construcción de ciudades del conocimiento: algunas reflexiones sobre la Sede Bogotá de la Universidad Nacional de Colombia. Paper presented at the La contribución 
de la educación superior al desarrollo político, social y económico de los países del Sur y las políticas para enfrentar los retos presentes y futuros, México.

Conti, A., \& Visentin, F. (2015a). A revealed preference analysis of PhD students' choices over employment outcomes. Research Policy, 44(10), 1931-1947. doi:http://dx.doi.org/10.10 16/j.respol.2015.06.009

Conti, A., \& Visentin, F. (2015b). Science and Engineering Ph.D. Students? Career Outcomes, by Gender. PLOS ONE, 10(8), e0133177. doi:10.1371/journal.pone.0133177

Enders, J. (2002). Serving Many Masters: The PhD on the Labour Market, the Everlasting Need of Inequality, and the Premature Death of Humboldt. Higher Education, 44, 493-517.

Evers, A., \& Sieverding, M. (2015). Academic career intention beyond the PhD: can the theory of planned behavior explain gender differences? Journal of Applied Social Psychology, 45(3), 158-172. doi:10.1111/jasp.12285

Fox, M. F., \& Stephan, P. (2001). Careers of Young Scientists: Preferences, Prospects and Realities by Gender and. Social Studies of Science, 31(1), 109-122.

Fritsch, N.-S. (2016). Patterns of career development and their role in the advancement of female faculty at Austrian universities: New roads to success? Higher Education, 72(5), 619635. doi:10.1007/s10734-015-9967-6

Gibbs, K. D., McGready, J., Bennett, J. C., \& Griffin, K. (2014). Biomedical Science Ph.D. Career Interest Patterns by Race/Ethnicity and Gender. PLOS ONE, 9(12), e114736. doi:10.1371/journal. pone. 0114736

Gómez, V., \& Celis, J. (2009). Sistema de aseguramiento de la calidad de la educación superior: consideraciones sobre la acreditación en Colombia. Revista Colombiana de Sociología, 32(1), 87-110.
Herrera, L., Baca, E., Aguilar, G., \& Nieto, M. (2013). Incorporación de Doctores a la Empresa: Un Análisis de los Factores Determinantes a Nivel Individual. Paper presented at the XV Congreso Latino Iberoamericano de Gestión Tecnológica, España.

Mangematin, V. (2000). PhD job market: professional trajectories and incentives during the PhD. Research Policy, 29, 741-756.

MEN-SNIES. (2016). Estadísticas de Educación Superior. Retrieved from Bogotá: http://www. mineducacion.gov.co/sistemasdeinformacion/1735/articles-212350_Estadisticas_de_ Educacion_Superior_.pdf

NSF. (2013). Survey of Earned Doctorates. July 1, 2012 to June 30, 2013. Retrieved from USA:

OCYT. (2016). Indicadores de ciencia y tecnología, Colombia 2015. Bogotá: Observatorio Colombiano de Ciencia y Tecnología.

OLE. (2016). Graduados de doctorado en ingeniería en Colombia según sexo (Vol. 2013). Bogotá: Ministerio de Educación Nacional.

Pineda, P. (2015). The Entrepreneurial Research University in Latin America. Global and Local Models in Chile and Colombia, 1950-2015. The United States: Palgrave Macmillan.

Roach, M., \& Sauermann, H. (2010). A taste for science? PhD scientists' academic orientation and self-selection into research careers in industry. Research Policy, 39, 422-434.

Roach, M., \& Sauermann, H. (2012). Founder or Joiner? The Interplay between Individual Preferences and Social-Contextual Influences in Shaping Entrepreneurial Interests. Paper presented at the DRUID Summer Conference, London.

Rubio, C., Celis, J., \& Gómez, F. (2007). Fortalecimiento de las capacidades investigativas del Sistema Nacional de Ciencia y Tecnología. Bogotá: COLCIENCIAS. 
Salmi, J. (2009). The Challenge of Establishing World-Class Universities. Washington: The World Bank.

Sauermann, H. (2005). Vocational choice: A decision making perspective. Journal of Vocational Behavior, 66, 273-303.

Sauermann, H. (2008). Individual incentives as drivers of innovative processes and performance. (Doctor of Philosophy), Duke University, USA.

Sauermann, H., \& Roach, M. (2012a). Science PhD career preferences: levels, changes, and advisor encouragement. PLOS ONE, 7(5), e36307.

Sauermann, H., \& Roach, M. (2012b). Taste for Science, Taste for Commercialization, and
Hybrid Scientists. Paper presented at the 34th DRUID Celebration Conference 2012, Denmark.

Sauermann, H., \& Roach, M. (2014). Not all scientists pay to be scientists: PhDs' preferences for publishing in industrial employment. Research Policy, 43, 32-47.

Stephan, P. (2012). How Economics Shapes Sciences. Cambridge, Massachusetss: Harvard University Press.

Wolfinger, N. H., Mason, M. A., \& Goulden, M. (2009). Stay in the Game: Gender, Family Formation and Alternative Trajectories in the Academic Life Course. Social Forces, 87(3), 1591-1621. doi:10.1353/sof.0.0182 